\title{
Potential Theory for Boundary Value Problems on finite networks
}

\author{
E. Bendito, A. Carmona*, A. M. Encinas and J.M. Gesto \\ Departament de Matemàtica Aplicada III \\ Universitat Politècnica de Catalunya. \\ 08034 Barcelona. Spain. \\ * e-mail:angeles.carmona@upc.edu
}

\begin{abstract}
We aim here at analyzing self-adjoint boundary value problems on finite networks associated with positive semi-definite Schrödinger operators. We see such operators as perturbations of the combinatorial Laplacian through 0-order terms that can be totally negative on a proper subset of the network. In addition, we prove that these discrete BVP have analogous properties to the ones of BVP for elliptic second order operators on Riemannian manifolds; for instance we study the existence and uniqueness of solutions and its variational formulation. Moreover, we will tackle a well-known problem in the framework of Potential Theory, the so-called condenser principle. Then, we make a generalization of the concept of effective resistance between two vertices of the network when a part of it is connected with a medium with different conductance, that in particular contains the case in which a part of the boundary is insulated. Finally, we characterize the Green function of some BVP in terms of effective resistances, which is usually known as the inverse resistive problem.
\end{abstract}

Keywords: Combinatorial Laplacian, Schrödinger operators, discrete boundary value problems, Green function, effective resistance.

\section{Introduction}

In this paper we analyze self-adjoint boundary value problems on finite networks associated with positive semi-definite Schrödinger operators. Among others, we treat general mixed boundary value problems that include the well-known Dirichlet and Neumann problems and also the Poisson equation. In the last years, these problems have deserved the attention of many researchers, see for instance $[1,3,4,5]$. The first of that papers is concerned with the general analysis of self-adjoint boundary value problems associated with non-negative perturbations of the combinatorial Laplacian and its associated Green functions from a Potential Theory point of view. 
The two last ones are mainly concerned with the inverse problem of identifying the conductivity function of the network, in terms of the boundary data.

A Schrödinger operator on a finite network is an operator of the form $\mathcal{L}_{q}=\mathcal{L}+q$, where $\mathcal{L}$ is the combinatorial Laplacian of the network and $q$ is a function on the vertex set. That function is usually known as ground-state since it represent that each vertex of the network is connected with a conductor medium with null potential. So, a Schrödinger operator can be seen as a perturbation of the combinatorial Laplacian. It is well-known that the energy associated with this operator is a Dirichlet form if and only if the ground state is non-negative, [7]. Some of the authors obtained in [3] a generalization of this result, when the ground state takes negative values, which was applied to the study of Dirichlet problems and Poisson equations. Here we extend the above results to the energy associated with general self-adjoint BVP. In particular, we show that any BVP has a unique solution provided that its associated energy is positive definite and we characterize when this happens in terms of the ground state. Moreover, we tackle the variational treatment of the self-adjoint BVP and we obtain the general version of the celebrated Dirichlet Principle.

In addition, we are concerned with the Condenser Principle, a classic topic in the framework of the Potential Theory associated with BVP. We extend the situation treated in [2], where only the case in which the ground state is null and a part of the boundary is insulated was considered. For that, we first tackle the natural extension, namely when the ground state is associated with a weight; which allows us to define the effective resistance with respect to this weight. As byproducts we obtain the Generalized Foster's Theorem that relates the total amount of the ratios between the conductances of the network and the effective conductances, see [9] for its usual formulation, and the expression of the Green function for the problem in which a single vertex is grounded in terms of the effective resistances. In its classical statement this expression is known as the inverse resistive problem and it has been considered for several author. The problem is the following: Let $(c(x, y))_{x, y \in V}$ denote the edge conductances of an electrical network, so that there is a resistor of $r_{x y}=1 / c(x, y)$ ohms between nodes $x$ and $y$. This uniquely determines the matrix $\left(R_{x y}\right)_{x, y \in V}$ of effective resistances, defined such that if a potential of $1 \mathrm{~V}$ is applied across nodes $x$ and $y$, a current of $1 / R_{x y}$ A will flow. Matrix $(c(x, y))_{x, y \in V}$ is called the resistive inverse of $\left(R_{x y}\right)_{x, y \in V}$. Coppersmith et al. [6] gave a simple but obscure four-step algorithm for computing the resistive inverse. After Ponzio gave a self-contained combinatorial explanation of this algorithm, [8]. In this work we prove an analogous result when more general cases are considered. To do that we consider the effective resistances, which can be obtained from the solution of condenser problems. Next we determine the Green function for the problem in terms of the effective resistances. Therefore, to obtain the inverse resistive it will suffice to invert the Green function and to complete this inverse so that it be the Laplacian of the network.

Finally, we study the case in which the energy is positive definite and we show that the Green function for the corresponding Robin problem can be also obtained as an inverse resistive of a suitable network. 


\section{Preliminaries}

Along the paper, $\Gamma=(V, E)$ denotes a simple, finite and connected graph without loops, with vertex set $V$ and edge set $E$. Two different vertices, $x, y \in V$, are called adjacent, which will be represented by $x \sim y$, if $\{x, y\} \in E$. Given $x, y \in V$, if $d(x, y)$ is the length of the shortest path joining $x$ and $y$ it is well-known that $d$ defines a distance on the graph.

Given a vertex subset $F \subset V$, we denote by $F^{c}$ its complementary in $V$ and we call boundary and closure of $F$, the sets $\delta(F)=\{x \in V: d(x, F)=1\}$ and $\bar{F}=F \cup \delta(F)$, respectively. Clearly, $\bar{F}=\{x \in V: d(x, F) \leq 1\}$.

The sets of functions and non-negative functions on $V$ are denoted by $\mathcal{C}(V)$ and $\mathcal{C}^{+}(V)$ respectively. If $u \in \mathcal{C}(V)$, its support is given by $\operatorname{supp}(u)=\{x \in V: u(x) \neq 0\}$. Moreover, if $F$ is a non empty subset of $V$, its characteristic function is denoted by $\chi_{F}$ and we can consider the sets $\mathcal{C}(F)=\{u \in \mathcal{C}(V): \operatorname{supp}(u) \subset F\}$ and $\mathcal{C}^{+}(F)=\mathcal{C}(F) \cap \mathcal{C}^{+}(V)$. For any $u \in \mathcal{C}(F)$, we

denote by $\int_{F} u(x) d x$ or simply by $\int_{F} u d x$ the value $\sum_{x \in F} u(x)$. We call weight on $F$ any function $\sigma \in \mathcal{C}^{+}(F)$ such that $\operatorname{supp}(\sigma)=F$. The set of weights on $F$ is denoted by $\mathcal{C}^{*}(F)$.

We call conductance on $\Gamma$ a function $c: V \times V \longrightarrow \mathbb{R}^{+}$such that $c(x, y)>0$ iff $x \sim y$. We call network any pair $(\Gamma, c)$, where $c$ is a conductance on $\Gamma$. In what follows we consider fixed the network $(\Gamma, c)$ and we refer to it simply by $\Gamma$.

The combinatorial Laplacian or simply the Laplacian of the network $\Gamma$ is the linear operator $\mathcal{L}: \mathcal{C}(V) \longrightarrow \mathcal{C}(V)$ that assigns to each $u \in \mathcal{C}(V)$ the function

$$
\mathcal{L}(u)(x)=\int_{V} c(x, y)(u(x)-u(y)) d y, \quad x \in V .
$$

If $F$ is a proper subset of $V$, for each $u \in \mathcal{C}(\bar{F})$ we define the normal derivative of $u$ as the function in $\mathcal{C}(\delta(F))$ given by

$$
\left(\frac{\partial u}{\partial \mathrm{n}_{\mathrm{F}}}\right)(x)=\int_{F} c(x, y)(u(x)-u(y)) d y, \text { for any } x \in \delta(F) .
$$

The relation between the values of the Laplacian on $F$ and the values of the normal derivative at $\delta(F)$ is given by the First Green Identity, proved in [1]

$$
\int_{F} v \mathcal{L}(u) d x=\frac{1}{2} \int_{\bar{F}} \int_{\bar{F}} c_{F}(x, y)(u(x)-u(y))(v(x)-v(y)) d x d y-\int_{\delta(F)} v \frac{\partial u}{\partial \mathrm{n}_{\mathrm{F}}} d x, \quad u, v \in \mathcal{C}(\bar{F}),
$$

where $c_{F}=c \cdot \chi_{(F \times F) \backslash(\delta(F) \times \delta(F))}$. A direct consequence of the above identity is the so-called Second Green Identity

$$
\int_{F}(v \mathcal{L}(u)-u \mathcal{L}(v)) d x=\int_{\delta(F)}\left(u \frac{\partial v}{\partial \mathrm{n}_{\mathrm{F}}}-v \frac{\partial u}{\partial \mathrm{n}_{\mathrm{F}}}\right) d x, \text { for all } u, v \in \mathcal{C}(\bar{F})
$$


When $F=V$ the above identity tell us that the combinatorial Laplacian is a self-adjoint operator and that $\int_{V} \mathcal{L}(u) d x=0$ for any $u \in \mathcal{C}(V)$. Moreover, since $\Gamma$ is connected $\mathcal{L}(u)=0$ iff $u$ is a constant function.

Given $q \in \mathcal{C}(V)$ the Schrödinger operator on $\Gamma$ with ground state $q$ is the linear operator $\mathcal{L}_{q}: \mathcal{C}(V) \longrightarrow \mathcal{C}(V)$ that assigns to each $u \in \mathcal{C}(V)$ the function $\mathcal{L}_{q}(u)=\mathcal{L}(u)+q u$.

\section{Self-adjoint Boundary Value Problems}

In this section we study different type of boundary value problems associated with the Schrödinger operator with ground state $q$. Given a non-empty subset $F \subset V, \delta(F)=H_{1} \cup H_{2}$, where $H_{1} \cap H_{2}=\emptyset$ and functions $g \in \mathcal{C}(F), g_{1} \in \mathcal{C}\left(H_{1}\right), g_{2} \in \mathcal{C}\left(H_{2}\right)$, a boundary value problem on $F$ consists on finding $u \in \mathcal{C}(\bar{F})$ such that

$$
\mathcal{L}_{q}(u)=g \text { on } F, \quad \frac{\partial u}{\partial \mathrm{n}_{\mathrm{F}}}+q u=g_{1} \text { on } H_{1} \quad \text { and } \quad u=g_{2} \text { on } H_{2} .
$$

In addition, the associated homogeneous boundary value problem consists on finding $u \in \mathcal{C}(\bar{F})$ such that $\mathcal{L}_{q}(u)=0$ on $F, \frac{\partial u}{\partial \mathrm{n}_{\mathrm{F}}}+q u=0$ on $H_{1}$ and $u=0$ on $H_{2}$.

The Green Identity implies that the boundary value problem (3) is self-adjoint in the sense that $\int_{F} v \mathcal{L}_{q}(u) d x=\int_{F} u \mathcal{L}_{q}(v) d x$ for all $u, v \in \mathcal{C}\left(F \cup H_{1}\right)$ verifying that $\frac{\partial u}{\partial \mathrm{n}_{\mathrm{F}}}+q u=\frac{\partial v}{\partial \mathrm{n}_{\mathrm{F}}}+q v=0$ on $H_{1}$.

Problem (3) is generically known as a mixed Dirichlet-Robin problem and summarizes the different boundary value problems that appear in the literature with the following proper names:

(i) Dirichlet problem: $\emptyset \neq H_{2}=\delta(F)$ and hence $H_{1}=\emptyset$.

(ii) Robin problem: $\emptyset \neq H_{1}=\delta(F)$ and $q \neq 0$ on $H_{1}$.

(iii) Neumann problem: $\emptyset \neq H_{1}=\delta(F)$ and $q=0$ on $H_{1}$.

(iv) Mixed Dirichlet-Neumann problem: $H_{1}, H_{2} \neq \emptyset$ and $q=0$ on $H_{1}$.

(v) Poisson equation on $V: F=V$.

The study of the boundary value problem (3) when $q \in \mathcal{C}^{+}(V)$ has been extensively treated, see for instance $[1,4,5]$ where the existence and uniqueness of solutions was established, whereas the analysis for Dirichlet Problem and Poisson equation in the case in which when $q$ can take negative value has been analyzed in [3]. In this work we extend the above results for the selfadjoint boundary value problem (3). 
Proposition 3.1 (Fredholm Alternative) Given $g \in \mathcal{C}(F), g_{1} \in \mathcal{C}\left(H_{1}\right), g_{2} \in \mathcal{C}\left(H_{2}\right)$, the boundary value problem

$$
\mathcal{L}_{q}(u)=g \quad \text { on } F, \quad \frac{\partial u}{\partial \mathrm{n}_{\mathrm{F}}}+q u=g_{1} \quad \text { on } H_{1} \quad \text { and } \quad u=g_{2} \text { on } H_{2}
$$

has solution iff for any $v \in \mathcal{C}(\bar{F})$ solution of the homogeneous problem it is verified

$$
\int_{F} g v d x+\int_{H_{1}} g_{1} v d x=\int_{H_{2}} g_{2} \frac{\partial v}{\partial \mathrm{n}_{F}} d x
$$

In addition, when the above condition holds, then there exists a unique $u \in \mathcal{C}(\bar{F})$ solution of the boundary value problem such that $\int_{\bar{F}} u v d x=0$, for any $v \in \mathcal{C}(\bar{F})$ solution of the homogeneous problem.

Proof. First observe that problem (3) is equivalent to the boundary value problem

$$
\mathcal{L}_{q}(u)=g-\mathcal{L}_{q}\left(g_{2}\right) \text { on } F, \frac{\partial u}{\partial \mathrm{n}_{\mathrm{F}}}+q u=g_{1} \text { on } H_{1} \text { and } u=0 \text { on } H_{2}
$$

in the sense that $u$ is a solution of this problem iff $u+g_{2}$ is a solution of (3).

Consider now the linear operator $\mathcal{F}: \mathcal{C}\left(F \cup H_{1}\right) \longrightarrow \mathcal{C}\left(F \cup H_{1}\right)$ defined as $\mathcal{F}(u)=\mathcal{L}_{q}(u)$ on $F$ and $\mathcal{F}(u)=\frac{\partial u}{\partial \mathrm{n}_{\mathrm{F}}}+q u$ on $H_{1}$. If $\mathcal{V}$ denotes the space of solutions of the homogeneous problem, then $\operatorname{ker} \mathcal{F}=\mathcal{V}$.

From the Second Green Identity, we get that $\int_{F \cup H_{1}} v \mathcal{F}(u) d x=\int_{F \cup H_{1}} u \mathcal{F}(v) d x$; that is, $\mathcal{F}$ is self-adjoint and hence $\operatorname{Img} \mathcal{F}=\mathcal{V}^{\perp}$, using the classical Fredholm Alternative. Consequently problem (3) has a solution iff the function $\tilde{g} \in \mathcal{C}\left(F \cup H_{1}\right)$ given by $\tilde{g}=g-\mathcal{L}_{q}\left(g_{2}\right)$ on $F$ and $\tilde{g}=g_{1}$ on $H_{1}$ verifies that

$0=\int_{F \cup H_{1}} \tilde{g} v d x=\int_{F} g v d x+\int_{H_{1}} g_{1} v d x-\int_{F} v \mathcal{L}_{q}\left(g_{2}\right) d x=\int_{F} g v d x+\int_{H_{1}} g_{1} v d x-\int_{H_{2}} g_{2} \frac{\partial v}{\partial \mathrm{n}_{\mathrm{F}}} d x$,

for any $v \in \mathcal{V}$. Finally, the Fredholm Alternative also establishes that when the necessary and sufficient condition are attained there exists a unique $w \in \mathcal{V}^{\perp}$ such that $\mathcal{F}(w)=\tilde{g}$. Therefore, $u=w+g_{2}$ is the unique solution of problem (3) such that for any $v \in \mathcal{V}$

$$
\int_{\bar{F}} u v d \nu=\int_{F \cup H_{1}} u v d \nu=\int_{F \cup H_{1}} w v d \nu=0,
$$

since $v=0$ on $H_{2}$ and $g_{2}=0$ on $F \cup H_{1}$.

Fredholm Alternative establishes that the existence of solution of problem (3) for any data $g, g_{1}$ and $g_{2}$ is equivalent to the uniqueness of solution and hence it is equivalent to the fact 
that the homogeneous problem has $v=0$ as its unique solution. So, applying the First Green Identity, if $v \in \mathcal{V}$

$$
0=\int_{F} v \mathcal{L}_{q}(v) d x=\frac{1}{2} \int_{\bar{F}} \int_{\bar{F}} c_{F}(x, y)(v(x)-v(y))^{2} d x d y+\int_{\bar{F}} q v^{2} d x
$$

and hence uniqueness is equivalent to be $v=0$ the unique solution of the above equality.

The above equality leads to define the energy associated with Problem (3) as the symmetric bilinear form $\mathcal{E}_{q}^{F}: \mathcal{C}(\bar{F}) \times \mathcal{C}(\bar{F}) \longrightarrow \mathbb{R}$ given for any $u, v \in \mathcal{C}(\bar{F})$ by

$$
\mathcal{E}_{q}^{F}(u, v)=\frac{1}{2} \int_{\bar{F}} \int_{\bar{F}} c_{F}(x, y)(u(x)-u(y))(v(x)-v(y)) d x d y+\int_{\bar{F}} q u v d x .
$$

A sufficient condition so that the homogeneous problem associated with (3) have $v=0$ as its unique solution is that the energy be positive definite. Next, we characterize when this property is achieved. To do this, it will be useful to introduce for any weight $\sigma$ on $\bar{F}$ the so-called ground state associated with $\sigma$ as $q_{\sigma}=-\frac{1}{\sigma} \mathcal{L}(\sigma)$ on $F, q_{\sigma}=-\frac{1}{\sigma} \frac{\partial \sigma}{\partial \mathrm{n}_{F}}$ on $\delta(F)$ and $q_{\sigma}=q$ otherwise. Clearly, if $\sigma \in \mathcal{C}^{*}(\bar{F})$ then for any $a>0, \mu=a \sigma \in \mathcal{C}^{*}(\bar{F})$ and moreover $q_{\mu}=q_{\sigma}$.

Observe that $q_{\sigma}=0$ iff $\sigma=a \chi_{\bar{F}}$, with $a>0$. More generally, if $\sigma \in \mathcal{C}^{*}(\bar{F})$, then tacking $v=\chi_{\bar{F}}$ in the second Green Identity we obtain that $\int_{\bar{F}} \sigma q_{\sigma}=0$, which implies that $q_{\sigma}$ must take positive and negative values, except when $\sigma=a \chi_{\bar{F}}, a>0$. Moreover, in [3] it was proved that $-\int_{\bar{F}} c_{F}(x, y) d y<q_{\sigma}(x)$ for any $x \in \bar{F}$ and also that when $H_{2} \neq \emptyset$, then it is possible to choose $\sigma \in \mathcal{C}^{*}(\bar{F})$ such that $q_{\sigma}(x)<0$ for any $x \in F \cup H_{1}$.

Proposition 3.2 The Energy $\mathcal{E}_{q}^{F}$ is positive semi-definite iff there exists $\sigma \in \mathcal{C}^{*}(\bar{F})$ such that $q \geq q_{\sigma}$. Moreover, it is not strictly definite iff $q=q_{\sigma}$, in which case $\mathcal{E}_{q}^{F}(v, v)=0$ iff $v=a \sigma$, $a \in \mathbb{R}$.

Proof. Consider the network $\Gamma_{F}=\left(\bar{F}, \bar{E}, c_{F}\right)$, where $\bar{E}=\left\{(x, y) \in E: c_{F}(x, y)>0\right\}$ and let $\overline{\mathcal{L}}$ its combinatorial Laplacian. Then, for any $u \in \mathcal{C}(\bar{F}), \overline{\mathcal{L}}(u)=\mathcal{L}(u)$ on $F$ and $\overline{\mathcal{L}}(u)=\frac{\partial u}{\partial \mathrm{n}_{F}}$ on $\delta(F)$. Moreover, $\mathcal{E}_{q}^{F}(u, u)=\int_{\bar{F}} u \overline{\mathcal{L}}(u) d x+\int_{\bar{F}} q u^{2} d x$ and hence the results follow by applying Proposition 3.3 and Corollary 3.4 of [3].

The next result establishes the fundamental result about the existence and uniqueness of solution for Problem (3) and about its variational formulation.

Proposition 3.3 (Dirichlet principle) Suppose that there exists $\sigma \in \mathcal{C}^{*}(\bar{F})$ such that $q \geq q_{\sigma}$. Given $g \in \mathcal{C}(F), g_{1} \in \mathcal{C}\left(H_{1}\right)$ and $g_{2} \in \mathcal{C}\left(H_{2}\right)$, consider the convex set $C_{g_{2}}=\{v \in \mathcal{C}(\bar{F}): v=$ 
$g_{2}$ on $\left.H_{2}\right\}$ and the quadratic functional $\mathcal{J}_{q}: \mathcal{C}(\bar{F}) \longrightarrow \mathbb{R}$ determined by the expression

$$
\mathcal{J}_{q}(u)=\frac{1}{2} \int_{\bar{F}} \int_{\bar{F}} c_{F}(x, y)(u(x)-u(y))^{2} d x d y+\int_{\bar{F}} q u^{2} d x-2 \int_{F} g u d x-2 \int_{H_{1}} g_{1} u d x .
$$

Then $u \in \mathcal{C}(\bar{F})$ is a solution of (3) iff $u$ minimizes $\mathcal{J}_{q}$ on $C_{g_{2}}$. Moreover, if it is not simultaneously true that $H_{2}=\emptyset$ and $q=q_{\sigma}$, then $\mathcal{J}_{q}$ has a unique minimum on $C_{g_{2}}$. Otherwise, $\mathcal{J}_{q}$ has a minimum iff $\int_{F} g \sigma d x+\int_{\delta(F)} g_{1} \sigma d x=0$. In this case, there exists a unique minimum $u \in \mathcal{C}(\bar{F})$ such that $\int_{\bar{F}} u \sigma d x=0$.

Proof. Observe first that $C_{g_{2}}=g_{2}+\mathcal{C}\left(F \cup H_{1}\right)$ and that for all $v \in \mathcal{C}\left(F \cup H_{1}\right)$ we get $\mathcal{J}_{q}(v)=\mathcal{E}_{q}^{F}(v, v)-2 \int_{F} g v d x-2 \int_{H_{1}} g_{1} v d x$. Keeping in mind, that $q \geq q_{\sigma}$, we get that $\mathcal{J}_{q}$ is a convex functional on $\mathcal{C}\left(F \cup H_{1}\right)$ and hence on $C_{g_{2}}$. Moreover, it is an strictly convex functional iff it is not simultaneously true that $H_{2}=\emptyset$ and $q=q_{\sigma}$ and then $\mathcal{J}_{q}$ has a unique minimum on $C_{g_{2}}$.

On the other hand, when $H_{2}=\emptyset$ and $q=q_{\sigma}$ simultaneously the minima of $\mathcal{J}_{q}$ are characterized by the Euler identity: $\mathcal{E}_{q}^{F}(u, v)=\int_{F} g v d x+\int_{H_{1}} g_{1} v d x$, for all $v \in \mathcal{C}(\bar{F})$. Since in this case $\mathcal{E}_{q}^{F}(u, \sigma)=0$ for all $u \in \mathcal{C}(\bar{F})$, necessarily $g$ and $g_{1}$ must satisfy that $\int_{F} g \sigma d x+\int_{H_{1}} g_{1} \sigma d x=0$. Moreover, if this condition holds and $\mathcal{V}$ denotes the vector subspace generated by $\sigma$, then $u \in \mathcal{V}^{\perp}$ minimizes $\mathcal{J}_{q}$ on $\mathcal{V}^{\perp}$ iff u minimizes $\mathcal{J}_{q}$ on $\mathcal{C}(\bar{F})$ and the existence of minimum follows since $\mathcal{J}_{q}$ is strictly convex on $\mathcal{V}^{\perp}$. In any case, the equations described in (3) are the Euler-Lagrange identities for the corresponding minimization problem.

The following result is an extension of the monotonicity property of the Schrödinger operator in the case $q \geq q_{\sigma}$ that was proved in [3].

Proposition 3.4 Suppose that $q \geq q_{\sigma}$ and that it is not simultaneously true that $H_{2}=\emptyset$ and $q=q_{\sigma}$. If $u \in \mathcal{C}(\bar{F})$ verifies that $\mathcal{L}_{q}(u) \geq 0$ on $F, \frac{\partial u}{\partial \mathrm{n}_{F}}+q u \geq 0$ on $H_{1}$ and $u \geq 0$ on $H_{2}$, then $u \in \mathcal{C}^{+}(\bar{F})$.

Proof. Consider again the network $\Gamma_{F}=\left(\bar{F}, \bar{E}, c_{F}\right)$, where $\bar{E}=\left\{(x, y) \in E: c_{F}(x, y)>0\right\}$ and let $\overline{\mathcal{L}}$ its combinatorial Laplacian. Then, if $u \in \mathcal{C}(\bar{F})$ verifies the hypotheses, $\overline{\mathcal{L}}(u) \geq 0$ on $F \cup H_{1}$ and the conclusion follows by applying Proposition 4.1 in [3].

Suppose that there exists $\sigma \in \mathcal{C}^{*}(\bar{F})$ such that $q \geq q_{\sigma}$ and it is not simultaneously true that $H_{2}=\emptyset$ and $q=q_{\sigma}$. The Green operator associated with Problem (3) is the linear operator 
$\mathcal{G}_{q}^{F}: \mathcal{C}(F) \longrightarrow \mathcal{C}(\bar{F})$ that assigns to any $g \in \mathcal{C}(F)$ the unique solution of the boundary value problem $\mathcal{L}_{q}(u)=g$ on $F, \frac{\partial u}{\partial \mathrm{n}_{F}}+q u=0$ on $H_{1}$ and $u=0$ on $H_{2}$. Moreover, we define the Green function associated with Problem (3) as the function $G_{q}^{F}: \bar{F} \times F \longrightarrow \mathbb{R}$ that assigns to any $y \in F$ and any $x \in \bar{F}$ the value $G_{q}^{F}(x, y)=\mathcal{G}_{q}^{F}\left(\varepsilon_{y}\right)(x)$, where $\varepsilon_{y}$ stands for the Dirac function at $y$. So, for any $g \in \mathcal{C}(F)$ it is verified that $\mathcal{G}_{q}^{F}(g)(x)=\int_{F} G_{q}^{F}(x, y) g(y) d y$. Finally, let us remark that from the above proposition $G_{q}^{F} \geq 0$ and moreover $G_{q}^{F}(x, y)=G_{q}^{F}(y, x)$ for any $x, y \in F$, since the boundary value problem (3) is self-adjoint.

\section{The Condenser principle}

In this section we obtain a generalization of the well-known Condenser Principle. From no on we suppose that there exists $\sigma \in \mathcal{C}(\bar{F})$ such that $q \geq q_{\sigma}$. Given a non-empty subset $F \subset V$, suppose that $\delta(F)=H_{1} \cup\{x\} \cup\{y\}$, where $x, y \notin H_{1}$ and $x \neq y$. The generalized Condenser Problem consists in the following mixed boundary value problem

$$
\mathcal{L}_{q}(u)=0 \quad \text { on } F, \quad \frac{\partial u}{\partial \mathrm{n}_{\mathrm{F}}}+q u=0 \text { on } H_{1}, \quad u(x)=\sigma(x) \text { and } \quad u(y)=0 .
$$

Proposition 4.1 (Condenser Principle) If $u \in \mathcal{C}(\bar{F})$ is the unique solution of the Condenser Problem (5), then $0 \leq u \leq \sigma$ on $V$.

Proof. The positiveness of $u$ follows directly from Proposition 3.4. Moreover, if $v=\sigma-u$ then $\mathcal{L}_{q}(v)=\sigma\left(q-q_{\sigma}\right) \geq 0$ on $F, \frac{\partial v}{\partial \mathrm{n}_{\mathrm{F}}}+q u=\sigma\left(q-q_{\sigma}\right) \geq 0$ on $H_{1}, \quad v(x)=0$ and $v(y)=\sigma(y)$. Therefore, applying again Proposition $3.4, v \geq 0$.

Under the hypotheses of the above proposition, $\bar{F}$ is called condenser with source and sink $x$ and $y$, respectively when $H_{1}$ is connected with a medium of conductivity $q$. Moreover, the above boundary value problem is called the condenser problem corresponding to $\bar{F}$.

Next we introduce a concept that is closely related with the condenser problem in the case $q=q_{\sigma}$, namely the effective resistance between $x$ and $y$ when a subset of the network, $D$, is connected with a medium of conductivity $q_{\sigma}$. Fixed a weight $\sigma \in \mathcal{C}^{*}(V)$ and the set $D \subset V$, consider for any $x, y \notin D$ with $x \neq y$, the unique solution $u \in \mathcal{C}(V)$ of the boundary value problem

$$
\mathcal{L}_{q_{\sigma}}(u)=0 \text { on } D^{c} \backslash\{x, y\}, \quad \frac{\partial u}{\partial \mathrm{n}_{D^{c}}}+q_{\sigma} u=0 \quad \text { on } \quad D, \quad u(x)=\sigma(x) \text { and } \quad u(y)=0 .
$$

The effective conductance between $x, y$ relative to $D$ with respect to $\sigma$, is defined as the value $C_{\sigma}^{D}(x, y)=\mathcal{E}_{q_{\sigma}}^{D^{c}}(u, u)$. Clearly, $C_{\sigma}^{D}(x, y)>0$, otherwise, $u=a \sigma$ and hence $u$ can not verify 
$u(y)=0$ and $u(x)=\sigma(x)$ simultaneously. In addition, it is verified that

$$
C_{\sigma}^{D}(x, y)=\int_{D^{c}} u \mathcal{L}_{q_{\sigma}}(u) d x+\int_{D} u\left(\frac{\partial u}{\partial \mathrm{n}_{D^{c}}}+q_{\sigma} u\right) d x=\sigma(x) \mathcal{L}_{q_{\sigma}}(u)(x)=-\sigma(y) \mathcal{L}_{q_{\sigma}}(u)(y) .
$$

The effective resistance between $x, y$ relative to $D$ with respect to $\sigma$, is defined as the value $R_{\sigma}^{D}(x, y)=C_{\sigma}^{D}(x, y)^{-1}$. The effective conductance, and hence the effective resistance, is a symmetric set function, that is, $C_{\sigma}^{D}(x, y)=C_{\sigma}^{D}(y, x)$ since $\mathcal{E}_{q_{\sigma}}^{D^{c}}(u, u)=\mathcal{E}_{q_{\sigma}}^{D^{c}}(\sigma-u, \sigma-u)$. So, it is irrelevant which vertex acts as the source and which one acts as the sink. On the other hand, applying the Dirichlet Principle we obtain that

$$
C_{\sigma}^{D}(x, y)=\min \left\{\mathcal{E}_{q_{\sigma}}^{D^{c}}(v, v): v(x)=\sigma(x) \text { and } v(y)=0\right\} .
$$

Proposition 4.2 If for any $z \notin D, \nu_{z}^{D} \in \mathcal{C}(V)$ denotes the unique solution of the problem

$$
\mathcal{L}_{q_{\sigma}}\left(\nu_{z}^{D}\right)=1 \text { on } D^{c} \backslash\{z\}, \quad \frac{\partial \nu_{z}^{D}}{\partial \mathrm{n}_{D^{c}}}+q_{\sigma} \nu_{z}^{D}=0 \text { on } D \text { and } \nu_{z}^{D}(z)=0,
$$

then the function

$$
u=\frac{\sigma(x)}{\sigma(y) \nu_{y}^{D}(x)+\nu_{x}^{D}(y) \sigma(x)}\left(\sigma(y) \nu_{y}^{D}-\sigma(y) \nu_{x}^{D}+\nu_{x}^{D}(y) \sigma\right)
$$

is the unique solution of the boundary value problem (6). In addition,

$$
R_{\sigma}^{D}(x, y)=\left(\int_{D^{c}} \sigma d x\right)^{-1}\left(\frac{\nu_{y}^{D}(x)}{\sigma(x)}+\frac{\nu_{x}^{D}(y)}{\sigma(y)}\right) .
$$

Proof. If $v=\sigma(y) \nu_{y}^{D}-\sigma(y) \nu_{x}^{D}+\nu_{x}^{D}(y) \sigma$, then a direct evaluation gives

$$
\mathcal{L}_{q_{\sigma}}(v)=0 \text { on } D^{c} \backslash\{x, y\}, \quad \frac{\partial v}{\partial \mathrm{n}_{D^{c}}}+q_{\sigma} v=0 \text { on } D \text { and } \quad v(y)=0 .
$$

Moreover $v(x)=\sigma(y) \nu_{y}^{D}(x)-\sigma(y) \nu_{x}^{D}(x)+\nu_{x}^{D}(y) \sigma(x)=\sigma(y) \nu_{y}^{D}(x)+\nu_{x}^{D}(y) \sigma(x)$, which implies that $u=\frac{\sigma(x) v}{\sigma(y) \nu_{y}^{D}(x)+\nu_{x}^{D}(y) \sigma(x)}$. On the other hand, applying the Identity (7), we get that

$$
C_{\sigma}^{D}(x, y)=\sigma(x) \mathcal{L}_{q_{\sigma}}(u)(x)=\frac{\sigma(x)^{2} \mathcal{L}_{q_{\sigma}}(v)(x)}{\sigma(y) \nu_{y}^{D}(x)+\nu_{x}^{D}(y) \sigma(x)} .
$$

Finally, tacking into account that $0=\int_{D^{c}} \sigma \mathcal{L}_{q_{\sigma}}\left(\nu_{x}^{D}\right) d x+\int_{D} \sigma\left(\frac{\partial \nu_{x}^{D}}{\partial \mathrm{n}_{D^{c}}}+q_{\sigma} \nu_{x}^{D}\right) d x$, we obtain that $0=\int_{D^{c}} \sigma \mathcal{L}_{q_{\sigma}}\left(\nu_{x}^{D}\right) d x=\int_{D^{c}} \sigma d x-\sigma(x)+\sigma(x) \mathcal{L}_{q_{\sigma}}\left(\nu_{x}^{D}\right)(x)$ and hence,

$$
\sigma(x) \mathcal{L}_{q_{\sigma}}(v)(x)=\sigma(x) \sigma(y) \mathcal{L}_{q_{\sigma}}\left(\nu_{y}^{D}\right)(x)-\sigma(x) \sigma(y) \mathcal{L}_{q_{\sigma}}\left(\nu_{x}^{D}\right)(x)=\sigma(y) \int_{D^{c}} \sigma d x
$$


which implies that

$$
C_{\sigma}^{D}(x, y)=\frac{\sigma(x) \sigma(y)}{\sigma(y) \nu_{y}^{D}(x)+\nu_{x}^{D}(y) \sigma(x)} \int_{D^{c}} \sigma d x
$$

and the last claim follows.

Observe that if for any $x \notin D$ we define $R_{\sigma}^{D}(x, x)=0$, then the above formula for the effective resistance between two vertices in $D^{c}$ is still valid for $y=x$. Now we can generalize a well-known result about the effective resistance.

Corollary 4.3 (Generalized Foster's Theorem) The following identity holds

$$
\int_{D^{c}} \int_{D^{c}} R_{\sigma}^{D}(x, y) c_{D^{c}}(x, y) \sigma(x) \sigma(y) d x d y=2(|V|-|D|-1) .
$$

Proof. From the expression of the effective resistance, we have that

$$
\sigma(x) \sigma(y) R_{\sigma}^{D}(x, y)=\left(\int_{D^{c}} \sigma d x\right)^{-1}\left(\sigma(y) \nu_{y}^{D}(x)+\sigma(x) \nu_{x}^{D}(y)\right) .
$$

On the other hand, tacking into account the symmetry of $c_{D^{c}}$ we get that

$$
\int_{D^{c}} \int_{D^{c}} \sigma(x) \nu_{x}^{D}(y) c_{D^{c}}(x, y) d x d y=\int_{D^{c}} \int_{D^{c}} \sigma(y) \nu_{y}^{D}(x) c_{D^{c}}(x, y) d x d y
$$

which implies that

$$
\int_{D^{c}} \int_{D^{c}} R_{\sigma}^{D}(x, y) c_{D^{c}}(x, y) \sigma(x) \sigma(y) d x d y=2\left(\int_{D^{c}} \sigma d x\right)^{-1} \int_{D^{c}} \sigma(x) \int_{D^{c}} \nu_{x}^{D}(y) c_{D^{c}}(x, y) d y d x .
$$

Finally, the result follows by keeping in main that for any $x \in D^{c}$

$$
\sigma(x) \int_{D^{c}} \nu_{x}^{D}(y) c_{D^{c}}(x, y) d y=\sigma(x) \mathcal{L}_{q_{\sigma}}\left(\nu_{x}^{D}\right)(x)=\int_{D^{c}} \sigma d x-\sigma(x) .
$$

Another well-known consequence of Proposition 4.2 establishes that when $q=q_{\sigma}$ for any $y \notin D$, the Green function for problem

$$
\mathcal{L}_{q_{\sigma}}(u)=f \text { on } D^{c} \backslash\{y\}, \quad \frac{\partial u}{\partial \mathrm{n}_{D^{c}}}+q_{\sigma} u=0 \text { on } D, \quad u(y)=0
$$

can be seen as an inverse resistive; i.e. can be expressed in terms of effective resistances.

Corollary 4.4 Given $x, y, z \notin D$ it is verified that

$$
G_{q_{\sigma}}^{D^{c} \backslash\{y\}}(z, x)=\frac{1}{2} \sigma(x) \sigma(z)\left(R_{\sigma}^{D}(x, y)+R_{\sigma}^{D}(z, y)-R_{\sigma}^{D}(z, x)\right) .
$$

In particular, the effective resistance determines a distance on $D^{c}$. 
Proof. First, observe that if $u$ is the solution of Problem (6), then Identity (7) implies that $\mathcal{L}_{q_{\sigma}}(u)=\frac{C_{\sigma}^{D}(x, y)}{\sigma}\left(\varepsilon_{x}-\varepsilon_{y}\right)$ on $D^{c}$ and hence $G_{q_{\sigma}}^{D^{c} \backslash\{y\}}(z, x)=R_{\sigma}^{D}(x, y) \sigma(x) u(z)$ for any $x \notin D$ and $z \in V$, that is

$$
G_{q_{\sigma}}^{D^{c} \backslash\{y\}}(z, x)=\left(\int_{D^{c}} \sigma d x\right)^{-1} \sigma(x) \sigma(z)\left(\frac{\nu_{y}^{D}(z)}{\sigma(z)}-\frac{\nu_{x}^{D}(z)}{\sigma(z)}+\frac{\nu_{x}^{D}(y)}{\sigma(y)}\right) .
$$

In particular, when $x, z \notin D$, then

$$
G_{q_{\sigma}}^{D^{c} \backslash\{y\}}(x, z)=\left(\int_{D^{c}} \sigma d x\right)^{-1} \sigma(x) \sigma(z)\left(\frac{\nu_{y}^{D}(x)}{\sigma(x)}-\frac{\nu_{z}^{D}(x)}{\sigma(x)}+\frac{\nu_{z}^{D}(y)}{\sigma(y)}\right)
$$

and the expression of the Green function is a consequence of its symmetry on $D^{c}$. In addition, the last conclusion is a direct consequence of being $G_{q_{\sigma}}^{D^{c} \backslash\{y\}}$ non-negative.

We finish this section by generalizing the above corollary to the case $q \geq q_{\sigma}$. Specifically, we prove that the Green function of the Robin boundary value problem

$$
\mathcal{L}_{q}(u)=f \text { on } D^{c}, \quad \frac{\partial u}{\partial \mathrm{n}_{D^{c}}}+q u=0 \text { on } D,
$$

can be seen as an inverse resistive relative to a new network. To do this, consider a new vertex $\hat{x} \notin V$, the set $\hat{V}=V \cup\{\hat{x}\}$ and $\hat{\sigma} \in \mathcal{C}^{*}(\hat{V})$ the weight on $\hat{V}$ defined as $\hat{\sigma}(x)=\sigma(x)$ when $x \in V$ and as $\sigma(\hat{x})=1$.

We consider the network $\hat{\Gamma}=(\hat{V}, \hat{E}, \hat{c})$ where $\hat{c}(x, y)=c(x, y)$ when $x, y \in V$ and $\hat{c}(\hat{x}, x)=$ $\sigma(x)\left(q(x)-q_{\sigma}(x)\right)$ for any $x \in V$. Therefore, $E$ is a proper subset of $\hat{E}$ and this also assures that $\hat{\Gamma}$ is connected. In addition, we denote by $\hat{\mathcal{L}}$ the combinatorial Laplacian of $\hat{\Gamma}$ and by $q_{\hat{\sigma}}$ the ground state associated with $\hat{\mathcal{L}}$ and $\hat{\sigma}$. The following result will be the key for our purposes.

Proposition 4.5 For any $u \in \mathcal{C}(\hat{V})$, it is verified that

$$
\hat{\mathcal{L}}(u)+q_{\hat{\sigma}} u=\mathcal{L}\left(u_{\left.\right|_{V}}\right)+q u-\left(q-q_{\hat{\sigma}}\right) u(\hat{x}) \text { on } V
$$

and

$$
\frac{\partial u}{\partial \mathrm{n}_{\hat{V} \backslash D}}+q_{\hat{\sigma}} u=\frac{\partial u}{\partial \mathrm{n}_{V \backslash D}}+q u-\left(q-q_{\hat{\sigma}}\right) u(\hat{x}) \text { on } D .
$$

In particular, if $u \in \mathcal{C}(V)$, then $\hat{\mathcal{L}}_{q_{\hat{\sigma}}}(u)=\mathcal{L}_{q}(u)$ on $V$ and $\frac{\partial u}{\partial \mathrm{n}_{\hat{V} \backslash D}}+q_{\hat{\sigma}} u=\frac{\partial u}{\partial \mathrm{n}_{V \backslash D}}+q u$ on $D$.

Proof. Given $u \in \mathcal{C}(\hat{V})$, we get that for any $x \in V$

$$
\hat{\mathcal{L}}(u)(x)=\mathcal{L}\left(u_{\left.\right|_{V}}\right)(x)+\hat{c}(x, \hat{x})(u(x)-u(\hat{x})) .
$$


In particular, tacking $u=\hat{\sigma}$ it is verified that $\hat{\mathcal{L}}(\hat{\sigma})(x)=\mathcal{L}(\sigma)(x)+\hat{c}(x, \hat{x})(\sigma(x)-1)$, which implies that $\hat{c}(x, \hat{x})=q_{\sigma}(x)-q_{\hat{\sigma}}(x)+\frac{\hat{c}(x, \hat{x})}{\sigma(x)}=q(x)-q_{\hat{\sigma}}(x)$ and the result follows substituting the value of $\hat{c}(\cdot, \hat{x})$ in the expression of $\hat{\mathcal{L}}(u)(x)$. The same reasoning works for the normal derivative.

Corollary 4.6 For all $x, y \notin D$ it is verified that

$$
G_{q}^{D^{c}}(x, y)=\frac{1}{2} \sigma(x) \sigma(y)\left(R_{\hat{\sigma}}^{D}(x, \hat{x})+R_{\hat{\sigma}}^{D}(y, \hat{x})-R_{\hat{\sigma}}^{D}(x, y)\right),
$$

where $R_{\hat{\sigma}}^{D}$ is the effective resistance relative to $D$ with respect to $\hat{\sigma}$ in the network $\hat{\Gamma}$.

Proof. Taking into account the above proposition, we get that $u \in \mathcal{C}(V)$ is the unique solution of the problem

$$
\mathcal{L}_{q}(u)=f \text { on } D^{c}, \quad \frac{\partial u}{\partial \mathrm{n}_{D^{c}}}+q u=0 \text { on } D
$$

iff it is the unique solution of the mixed problem

$$
\hat{\mathcal{L}}_{q_{\hat{\sigma}}}(u)=f \text { on } D^{c}, \quad \frac{\partial u}{\partial \mathrm{n}_{\hat{V} \backslash D}}+q_{\hat{\sigma}} u=0 \text { on } D \text { and } u(\hat{x})=0 .
$$

The result follows by applying Corollary 4.4 to the network $\hat{\Gamma}$ and considering $y=\hat{x}$.

\section{Acknowledgments}

This work has been partly supported by the Spanish Research Council (Comisión Interministerial de Ciencia y Tecnología,) under project BFM2003-06014.

\section{References}

[1] E. Bendito, A. Carmona and A. M. Encinas, Solving boundary value problems on networks using equilibrium measures, J. Funct. Anal. 171 (2000), 155-176.

[2] E. Bendito, A. Carmona and A. M. Encinas, Equilibrium measure, Poisson kernel and effective resistance on networks, in "Random Walks and Geometry", (V. Kaimanovich, K. Schmidt and W. Woess ed.), 363-376, Walter de Gruyter, 2004.

[3] E. Bendito, A. Carmona and A. M. Encinas, Potential Theory for Schrödinger operators on finite networks, Rev. Mat. Iberoamericana 21 (2005), 771-818.

[4] Bensoussan, A., Menaldi, J.L., Difference equations on weighted graphs. J. Convex Anal. 12 (2005), 13-44. 
[5] Chung, S-Y., Berenstein, C.A., $\omega$-harmonic functions and inverse conductivity problems on networks. SIAM J. Appl. Math. 65 (2005), 1200-1226.

[6] Coppersmith, D., Doyle, P., Raghavan, P. and Snir, M., Random walks on weighted graphs and applications to on-line algorithms. J. Assoc. Comput. Mach. 40 (1993), 421-453.

[7] J. Kigami, "Analysis on fractals", Cambridge Tracts in Math., vol. 143. Cambridge University Press, 2001.

[8] S. Ponzio, The combinatorics of effective resistances and resistive inverses, Inform. and Comput. 147 (1998), 209-223.

[9] P. Tetali, An extension of Foster's network theorem, Combin. Probab. Comput. 3 (1994), 421-427. 